\title{
Circadian phagocytic activity of neutrophils and its modulation by light ${ }^{1}$
}

\author{
Monica L. Hriscu \\ Institute of Public Health, Occupational Medicine Department, Cluj-Napoca, Romania
}

Received $8^{\text {th }}$ September 2004.

Revised $11^{\text {th }}$ October 2004.

Published online $16^{\text {th }}$ November 2004.

\begin{abstract}
Summary
The experimental data reviewed herein is aimed at an evaluation of the circadian dynamics of neutrophil phagocytosis (basal phagocytosis, phagocytic response, and adherence) in mice and rats and attempts to establish whether the functional pinealectomy accomplished through constant light exposure affects the level and/or circadian oscillation of the parameters. In both species, basal phagocytic activity appears as a periodic function, peaking in the second half of the night (03:00 04:00). Adherence to nylon fibres displays a circadian oscillation with two maxima (10:00 and 22:00). Under constant light, the mean phagocytic activity is lowered by $40 \%$, but its circadian amplitude increases, while the acrophase occurs earlier. Both the mean adherence level and its circadian variation are depressed, the latter to a larger extent. The amplitude and shape of the phagocytic response in non-immunised mice appears to depend on the time of the day when antigenic stimulation occurs, thus on the phase of the basal rhythm, drawing attention to the necessity for careful circadian scheduling of immunomodulatory interventions. The results suggest that rhythmic phagocytosis is part of the immune system's circadian oscillation, controlled by hypothalamic centres and susceptible to modulation under the impact of endogenous and exogenous factors, including melatonin and the light-dark cycle.
\end{abstract}

Keywords: phagocytosis - neutrophils - circadian rhythms - light - melatonin - hypothalamus

\section{INTRODUCTION}

The temporal modulation of the immune system is an eminently neuroimmune interaction. Neural structures receive the environmental information that plays the role of an entraining signal; also, neural elements are responsible for the generation and synchronisation of the rhythms. The external variable is transduced to an internal signal by the pulsatile neuro-endocrine secretion of pineal melatonin, which acts both upon neural regulatory centres and upon immuno-competent cells, either directly or via other humoral factors. Feedback and "lateral feedbacks" originate from each or any of these levels, reaching their own regulatory systems and other systems that in their turn oscillate. Interrelations of this type exist at all structural levels and are fundamental for the co-ordination of rhythmic processes in the organism.

In the mammalian circadian system, the main external stimulus is light. Photic information, received by the retina, is transmitted via the retinohypothalamic tract to the central oscillator of the system, the suprachiasmatic nucleus (SCN) (Pickard 1982). Several effector pathways originating in the internal clock head towards the

${ }^{1}$ Dedicated to the memory of Professor Ion Baciu (1921-2004) 
neighbouring hypothalamic nuclei (Swanson and Cowan 1975, Berk and Finkelstein 1981). The dorso-caudal periventricular efference reaches the paraventricular nucleus and, from there, via a monosynaptic pathway, to the sympathetic preganglionic neurons in the spinal intermediolateral nucleus, which innervate the pineal gland through the superior cervical ganglion (Pickard and Turek 1983). The environmental light-dark cycle is transduced to an internal, neuroendocrine signal, represented by the rhythmic secretion of pineal melatonin, which always occurs during the dark period (Arendt 1995). Melatonin acts upon specific effector areas of the brain, such as the hypophyseal pars tuberalis (PT). It also acts on the retina, modulating its photosensitivity, and upon the SCN. The density of the melatonin receptors, both in the $\mathrm{PT}$ and in the SCN, exhibits a circadian rhythm which is in inverse correlation with the melatonin oscillation (down-regulation), thus rendering the control mechanisms of the system even more complex (Vanecek 1998).

Melatonin synthesis exhibits a prominent circadian rhythm, low during day and high at night; the rhythm is endogenous, persisting in animals kept under constant dark, and is determined by the rhythmic activity of $\mathrm{N}$-acetyl-transferase (Klein and Weller 1970). In most species, the duration of the secretory pulse is directly related to the length of the dark period (Arendt 1995, 1986). Thus, melatonin acts as a clock signalling dark onset/offset, and, on a larger time scale, as a calendar, which indicates the season in accord with the duration of the night-time period. The fact that melatonin secretion is linked in a mandatory fashion with the dark span makes "night" an absolute notion, irrespective of the active period of one species or another. This implies further that the existing relationship between circadian parameters related to other activity types and melatonin cannot be the same in all species.

The effects of melatonin on the immune system may differ in relation to factors such as species, the sex and age of the organism, the maturation and physiological status of the immune system, the activation pathway, the examined parameter, the dose of melatonin and route of administration, circadian timing, the season, etc. (Conti and Maestroni 1994, Maestroni et al. 1987). Melatonin enhances the specific primary response - humoral and cellular (Maestroni et al. 1986, 1987), prevents apoptosis of thymocytes (Sainz et al. 1995), increases the number and activity of $\mathrm{NK}$ cells (Angeli et al. 1988, Gatti et al. 1990) and may activate in vitro macrophages (Morrey 1994, Barjavel et al. 1998) and neutrophils (Rodríguez et al. 1997, Pieri et al. 1998). In birds and in mice, melatonin appears also to have stimulatory effects on neutrophils in vivo (Rodríguez and Lea 1994, Rodríguez et al. 2001, Barriga et al. 2001).

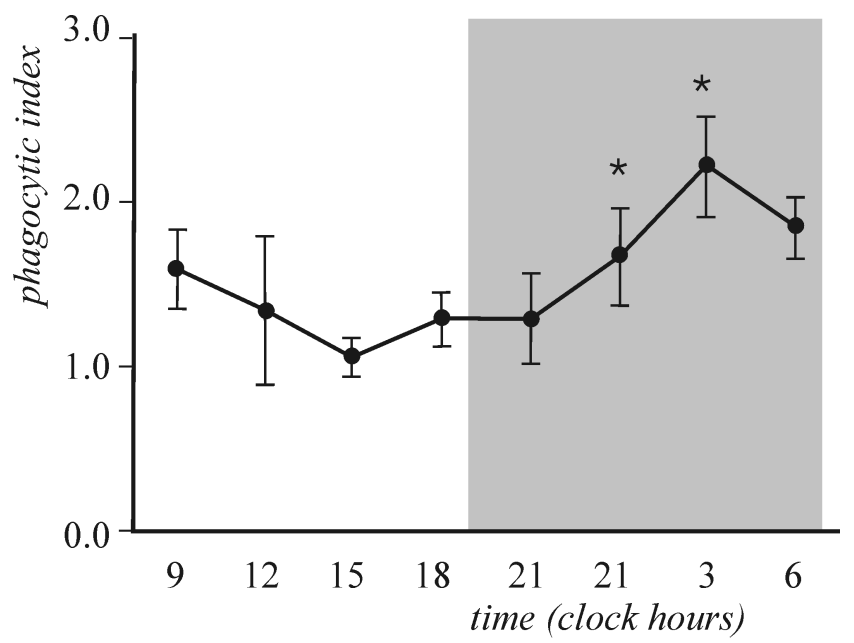

Fig. 1. The phagocytic index in mice over a 24-h period. Each value is the mean $\pm \mathrm{SD}$ of nine determinations. The shaded interval corresponds to the hours of darkness. ${ }^{*}$ statistically significant $(\mathrm{p}<0.05)$ as compared with values at all other time points

\section{EXPERIMENTAL BACKGROUND OF THE NERVOUS CONTROL OF PHAGOCYTOSIS}

Research initiated more than half a century ago and carried out by a team directed by I. Baciu, a member of the Romanian Academy, brought some of the earliest evidence that immune activity is under superior nervous control. These studies have shown that electric stimulation of the hypothalamic tubero-mammillary area (Benetato et al. 1945) or perfusion of the brain with bacteria-containing blood, using the "isolated head" model in dogs (Benetato et al. 1947), induce, via neural pathways, leukocytosis with neutrophilia and increase of the 
phagocytic activity, features which characterise the phagocytic response. This role of the tuberomammillary area was supported by the discovery that its electric stimulation induces medullar release of peptides with a phagocytosis-enhancing action (Baciu et al. 1961). Further confirmation was provided by Forni et al. (1983), who showed that radio frequency destruction of the tuberoinfundibular area permanently prevents NK cells activity in mice.

Studies regarding the role of the superior nervous centres (Baciu et al. 1988, 1994) revealed that the basal phagocytic activity of neutrophils in the blood of guinea pigs exhibits circadian oscillations, which are influenced by lesions of the preoptic and tubero-mammillary hypothalamus and also by changes in the lighting regimen.
In the species previously studied, the maximum phagocytic activity occurred either in the evening (guinea pigs, humans), or at night (ring dove). It was thus reasonable to assume that nocturnal melatonin secretion could play a role in supporting phagocytosis. The experimental data reviewed herein was obtained in order to evaluate the circadian dynamics of several parameters of phagocytosis (basal phagocytosis, phagocytic response, adherence of neutrophils) in mice and rats (Hriscu et al. 1998, Baciu et al. 2003). We attempted to find out if the functional pinealectomy accomplished through constant light exposure would affect the above-mentioned parameters and to discern to what extent their rhythmic oscillation is attributable to the entraining light-dark cycle (Hriscu and Saulea 2002).

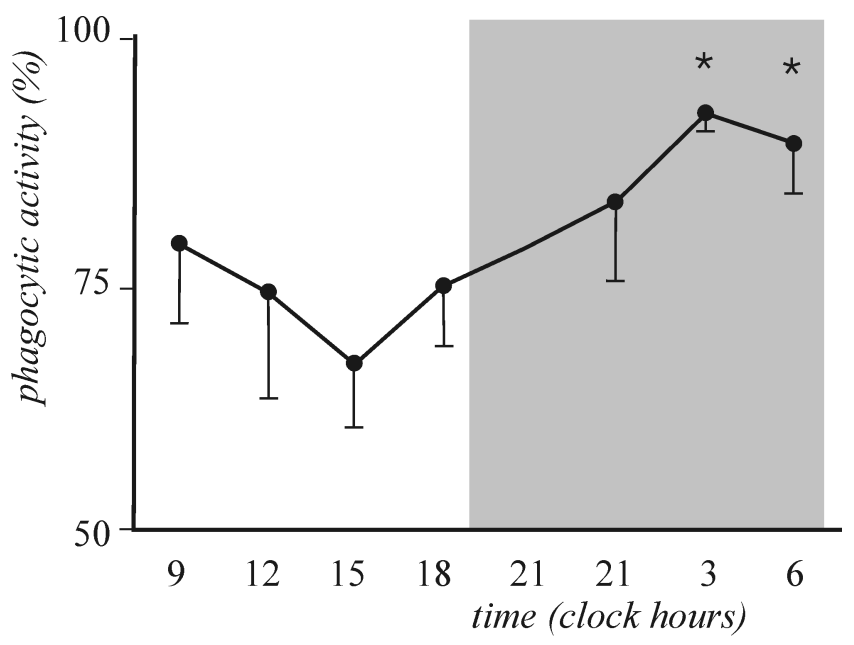

Fig. 2. The phagocytic activity in mice neutrophils over a 24-h period. Each value is the mean $\pm \mathrm{SD}$ of nine determinatios. The shaded band corresponds to the hours of darkness. * statistically significant $(\mathrm{p}<0.05)$ as compared with values at all other time points

\section{THE CIRCADIAN RHYTHM OF BASAL PHAGOCYTOSIS IN MICE}

NMRI mice, males of $20-25 \mathrm{~g}$ in weight, were kept under a natural light-dark alternation of $12: 12 \pm 0.5 \mathrm{~h}$, in a period of the year close to the autumnal equinox. Assessment of the phagocytic activity was made every three hours, according to a method described elsewhere (Hriscu et al. 1997). Briefly, heparinised whole blood was incubated for 30 min. at $37^{\circ} \mathrm{C}$ with an Escherichia coli suspension. The blood-bacteria mixture was spread and the neutrophils examined microscopically. Phagocytic activity was expressed as the number of bacilli engulfed by 100 neutrophils (phagocytic index, PI), and as the percent of neutrophils having engulfed at least one bacillus (phagocytic activity, PP). The phagocytic efficiency index was given by the PI/PP ratio. The circadian rhythms were characterised by the following parameters, obtained by a single cosinor analysis (Cornélissen and
Halberg 1994): mesor (rhythm-adjusted mean; when the interval of time between data sampling is constant, it equals the arithmetic mean), amplitude (A, semi-difference between the highest and the lowest value), acrophase ( $\Phi$, timing of overall high values), and $\mathrm{p}$, indicating the significance of the oscillation. A variability index of the rhythm (V), represented by the double amplitude as a percentage of the mesor, was also calculated.

The phagocytic index (Fig. 1) displayed a clearcut circadian curve, beginning to rise significantly after the onset of the dark phase and attaining its highest values after 03:00 (3.56h). A clear-cut decline was noticed after 06:00. Trough values were met at 15:00. Rhythm parameters were: $\mathrm{p}<0.05, \quad$ mesor $=1.534 \pm 0.0340, \quad \mathrm{~A}=0.460 \pm 0.0481$, $\Phi=3.56 \mathrm{~h} \pm 0.03, \mathrm{~V}=75 \%$.

T-test values for each time point vs. all the other points appeared to be significant for the 03:00h value: $\mathrm{p}_{(24: 00 \text { vs. } 03: 00)}<0.05, \mathrm{p}_{(03: 00 \text { vs. } 06: 00)}<0.05$ Other statistically significant differences were 
noticed between 09:00-2:00h (decrease) and 21:00-24:00 (increase), these data suggest that the acrophase has a rather peaked shape, with both the after dark increase and the decrease during the morning hours being fairly abrupt.
Phagocytic activity and phagocytic efficiency (Fig. 2, 3) displayed temporal structures similar to that of the phagocytic index, only with earlier acrophases $\quad(0.15 \mathrm{~h} \pm 0.26 \quad$ and $\quad 1.27 \mathrm{~h} \pm 0.02$, respectively).

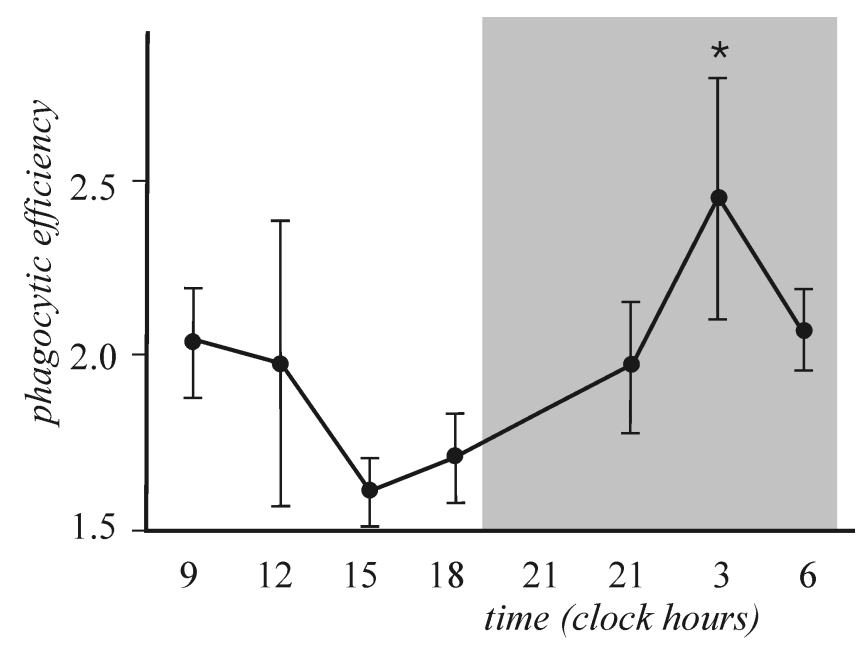

Fig. 3. The phagocytic efficiency in mice neutrophils over a 24-h period. Each value is the mean $\pm \mathrm{SD}$ of nine determinatios. The shaded band corresponds to the hours of darkness. * statistically significant $(\mathrm{p}<0.05)$ as compared with values at other time points

\section{INFLUENCE OF THE TIME OF CHALLENGE UPON THE MAGNITUDE OF THE PHAGOCYTIC RESPONSE}

NMRI mice, males weighing 20-25g, were kept for 14 days under an artificial light-dark 12:12 regimen (lights on between 09:00-21:00). The experiment took place in July. The phagocytic response was challenged by an i.p. injection of $10 \mu \mathrm{g} E$. coli lipopolysaccharide (LPS) $/ 25 \mathrm{~g}$ mouse. (E. coli 055:B5, Sigma Chemical Co.). The antigen was injected at one of the following hours: 10:00, 16:00, 22:00, and 04:00, different animals being used each time. The phagocytic index was tested before (=controls), and 4,8 and $24 \mathrm{~h}$ after the challenge.

The phagocytic response assumed different shapes according to the time of the day when the antigen encounter occurred and therefore with the phase of basal phagocytosis at the time of the challenge. At 10:00 and 16:00, the LPS injection induced a low amplitude response at $4 \mathrm{~h}$, followed by a slight depression at $8 \mathrm{~h}$ and a recovery at $24 \mathrm{~h}$, when the phagocytic index slightly exceeded its 4-h level. The variations were small, attaining a maximum of $10 \%$ of the initial value, and lacked statistical meaning.

When the antigenic challenge occurred at $22: 00 \mathrm{~h}$, an increase of about $30 \%(\mathrm{p}<0.05)$ of the phagocytic index value was noticed $4 \mathrm{~h}$ later. A marked depression $(\mathrm{p}<0.05)$ occurred 8 hours after the challenge, with the phagocytic index falling below the initial level. At 24h, the phagocytosis had recovered to a level slightly above baseline.

The LPS injection at 04:00 induced $4 \mathrm{~h}$ later an increase of more than $50 \%$ of the initial value $(51.8 \%, \mathrm{p}<0.05)$. After the depression at $8 \mathrm{~h}$, during which the level still retained an almost $10 \%$ elevation, the phagocityc index rose again, at $24 \mathrm{~h}$ surpassing the initial value by $36.8 \% \quad(p<0.05)$ (Fig. 4).

\section{PHAGOCYTIC ACTIVITY AND ADHERENCE OF NEUTROPHILS IN RATS SUBMITTED TO LIGHT-DARK ALTERNATION AND TO CONSTANT LIGHT}

The phagocytic activity on neutrophils in vivo represents a complex process which implies their adherence to the vascular endothelium, diapedesis to the extravascular compartment, directional migration in relation with chemotactic gradients and, finally, engulfing of the particulate material (phagocytosis proper) at the stimulus site (MacGregor et al. 1974). Adherence of neutrophils to the vascular walls, which actually initiates the process, has been studied less, in part also because of the technical difficulties of measuring adherence in experimental conditions. 


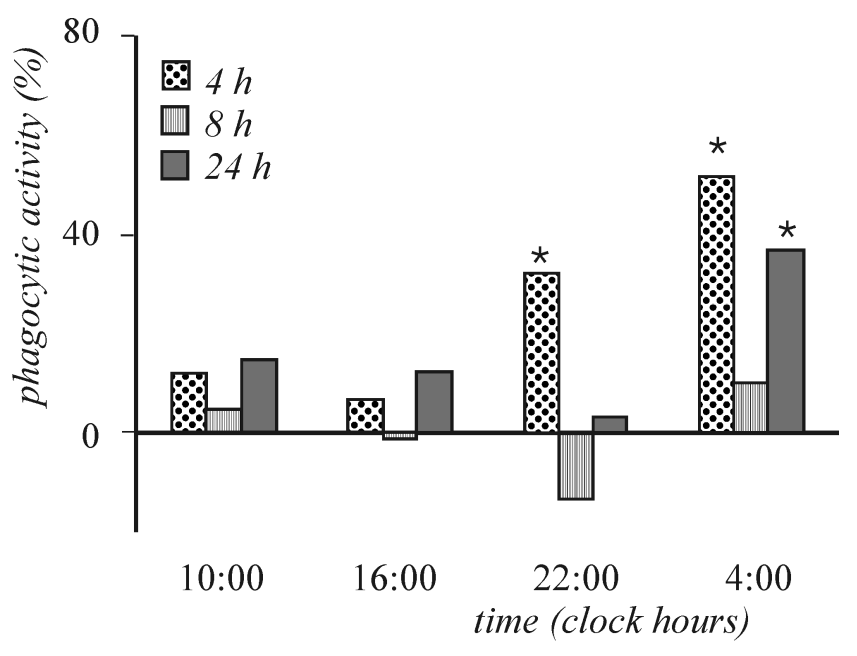

Fig. 4. Evolution of the phagocytic response challenged at different time points. The chart shows the difference in activity at 4,8 , and $24 \mathrm{~h}$ after the challenge against the initial phagocytic level (horizontal axis). * statistically significant as compared with controls $(\mathrm{p}<0.05)$

Nocturnal melatonin secretion can be suppressed by exposure to constant light, which produces a functional inhibition of the pineal (Arendt 1995, Vanecek 1998). In rats, one minute of fluorescent white light of 150 lux intensity is enough to induce rapid decline of NAT activity, thus decreasing plasmatic melatonin to an almost undetectable day level (Illnerova and Vanecek 1979).

Male Wistar rats, 180-200g, were maintained on an artificial light-dark 12:12 regimen (lights on between 07:00-19:00; white fluorescent light, 1500-2000lux; 5-15lux in the dark phase - LD group). A second group of rats (LL group) was subjected to constant light with the same characteristics as in the LD group. The experimental conditions were applied for 15 days before and during the testing period. Blood was collected every six hours during a 24 -h cycle. The total leukocyte count, and the numbers and percentages of neutrophils and lymphocytes were determined; the phagocytic index was assessed as previously described. A microassay system was employed for evaluation of the nylon fibre adherence of neutrophils in whole heparinised blood (Hriscu et al. 2003). According to MacGregor et al. (1978), the process of neutrophil adhesion to nylon fibres is similar to that of neutrophil adhesion to the vascular wall.

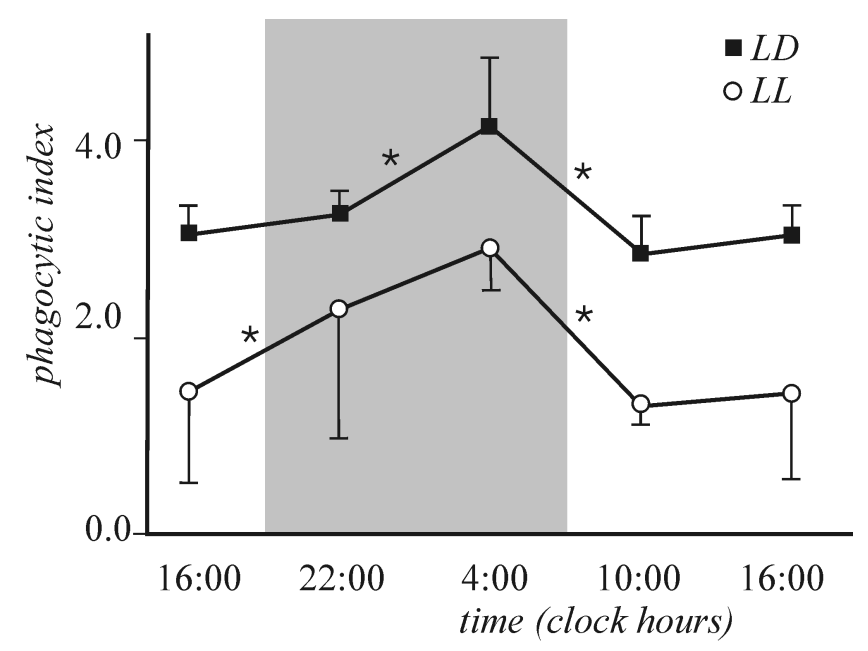

Fig. 5. The circadian phagocytic index in rats kept under 12h/12h light-dark conditions (LD) and under constant light $(L L)$. * statistically significant $(\mathrm{p}<0.05)$ as compared with adjacent values 
In the LD group, the phagocytic index exhibited a circadian rhythm with the acrophase at about 04:00 and the nadir at about 10:00. The phagocytic index remained low over the whole light period and in the first dark hours; a marked increase $(\mathrm{p}<0.05)$ occurred between 22:00 and 04:00. Characteristics of the rhythm were: mesor $=3.296 \pm 0.150$, amplitude $0.567 \pm 0.212$, acrophase $=2.39 \mathrm{~h}, \mathrm{p}>0.05$.

In the LL group, the circadian structure (timing of the acrophase and nadir) of the phagocytic index was largely preserved. The amplitude increased (by $25 \%$ larger than in LD); nevertheless, the phagocytic activity level appeared depressed, with the mesor only $60 \%$ of that in LD. In LL animals, the circadian curve assumed an ascending slope earlier in the afternoon: a significant increase occurred between 16:00 and 22:00 and the acrophase was advanced (1.43h) (Fig. 5).
The ability of neutrophils to adhere appeared to be a rhythmic function with two circadian maxima: the main peak at 10:00 and a lower one at 22:00. The rhythm had a mesor of $64.7 \pm 8.1$ and a double amplitude of $37.8 \pm 16.5$. The double amplitude of the secondary peak was about half of that of the main peak. The calculated acrophase was at $11.1 \mathrm{~h}$.

Constant light induced a slight decrease of the adherence ability (mesor about 10\% lower than in LD animals). The adherence was significantly depressed both during the circadian period of high values, at 10:00 $(\mathrm{p}<0.05)$, and in the evening at 22:00 $(\mathrm{p}<0.05)$. The acrophase occurred earlier (9.5h), and the amplitude diminished. The secondary increase at 22:00 almost disappeared, the rhythm thus losing its characteristic biphasic shape (Fig. 6).

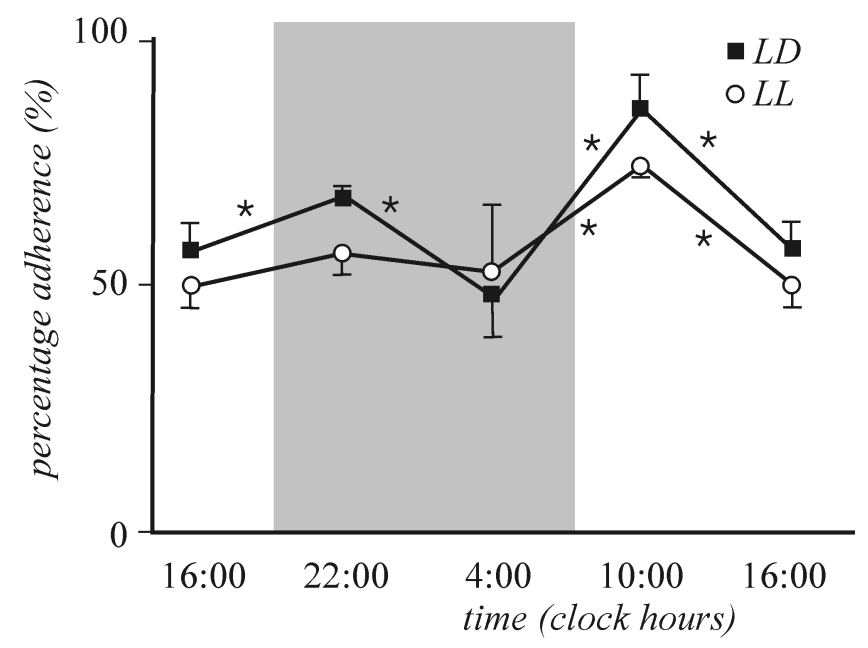

Fig. 6. The adherence rate of neutrophils over a $24 \mathrm{~h}$-cycle, in rats kept under $12 \mathrm{~h} / 12 \mathrm{~h}$ light-dark conditions (LD) and under constant light $(\mathrm{LL}){ }^{*}$ statistically significant $(\mathrm{p}<0.05)$ as compared with adjacent values

The LL regimen lowered the amplitude and the mesor of the leukocyte and the neutrophil rhythms by about $25 \%$. In both, however, the acrophase remained unaffected (at 10:00) (Fig. 7). The percentage of circulating neutrophils did not suffer any marked alterations; a slight overall decrease of about $10 \%$ was observed, except at the 22:00 time point.

\section{DISCUSSION}

The phagocytic activity of neutrophils is an eminently circadian function. In guinea pigs it attains maximal values soon after the light-dark transition (Baciu et al. 1988, 1994). Bongrand et al. (1988) reported a similar rhythm in humans, even though the oscillations lacked statistical significance, which could be expected in a relatively small group of only partially matched human subjects, as was then the case. In a species of birds (the ring dove, Streptopelia risoria), Rodríguez et al. (1999) described a circadian rhythm with the acrophase at about 04:00, concurrent with the maximum of plasma melatonin. We report here a statistically valid rhythm of phagocytosis in mice and rats. In these nocturnal rodents, the acrophase also occurred in the second half of the dark span (between 02:00 and 04:00). In mice, the nocturnal augmentation of the global phagocytic capacity is accountable both to an increase in the ability of neutrophils to engulf bacteria, as well as to the increasing number of active neutrophils; however, the factor with the highest circadian variability is the engulfing capacity (PI), with a variability index of $75 \%$, more than double that of the phagocytic activity. Nocturnal activation of phagocytosis begins after dark with an increase of the percentage of 
neutrophils displaying phagocytic activity, being followed in the next hours by a rise in the average number of bacteria engulfed by a neutrophil and reaching its maximum around 3 o'clock.

Both in humans and guinea pigs, on the one hand, and in mice and rats, on the other, the phagocytosis increases in the last hours of the active period. There is a concurrence in the late afternoon between the maxima of the neutrophil count and of their phagocytic activity in humans, (Bureau et al. 1987, Haus 1996), while in mice and rats the two peaks are about 6 hours apart: the phagocytosis peak is noticed in the second half of the dark span, while the highest number of circulating neutrophils occurs during the morning hours, as shown by different authors (Swoyer et al. 1987, Berger 1981) and by us. It can be suggested that phagocytosis in rodents and perhaps in other groups is linked to the type of activity of a specific species integrated with the circadian oscillations of the immune system (see the equivocal guinea pigand the diurnal human vs. the nocturnal mouse and rat). Our results are in good accordance with those reported by other authors (Sánchez et al. 2004) and partially concordant with those of Berger and Slapničková (2003), who described the phagocytic activity of rat neutrophils peaking in the morning hours (08:00) against small hydrophilic particles, while at midnight against yeast cells. These differences are possibly attributable to the different experimental designs employed, which in the mentioned study skipped the time points between 24:00 and 08:00.

The phagocytic response challenged by LPS injection appears to be different, in shape and amplitude, depending on the time of the day when the antigen encounter takes place, and its intensity depends on the phase of basal phagocytosis at the time of challenge. Antigenic stimulation in the light period, corresponding to a decreasing (10:00) or close to minimal (16:00) basal activity induces a long lasting phagocytic response, with the level 24 hours after stimulation, at least as high as the 4-h value. However, the overall increase is small, and thus doubtful in terms of biological significance. If the challenge is concurrent with the ascendant slope of the basal curve (22:00), the induced response is immediate and intense, though it is followed by an interval of marked depression and practically turns off completely in 24 hours. When the challenge coincides with the peak of basal phagocytosis, a high phagocytic response follows, which lasts at least over the next 24 hours.

The results of this study, as well as other experimental data (Hriscu 2003), suggest that the amplitude of the phagocytic response is conditioned not only by the moment of the day when the antigenic challenge takes place, but, at the same time, by the size of the antigen dose employed. A small antigen dose $(5 \mu \mathrm{gLPS} / 25 \mathrm{gb} / \mathrm{w})$ elicits a phagocytic response only during the high reactivity period, at night. A LPS dose twice as large is followed by enhanced phagocytosis at any time of the day, but the induced response is much higher in the dark period than during the day, thus confirming older results that suggested the existence of daily oscillations of immune reactivity (Halberg 1960), which can be modified through manipulations of the light-dark cycle (Pownall et al. 1979). From a practical perspective, these results draw attention to the necessity for a careful circadian planning of immunomodulatory interventions, either experimental or therapeutic, as the physiologic oscillations of the immune system may induce very different effects depending on the circadian moment of the intervention.

An argument for the circadian rhythmicity of the immune responsiveness is the fact that the depression of phagocytosis which follows after bilateral lesion of the anterior hypothalamic (AHA) and preoptic area in guinea pigs is most marked around the dark-light transition, at a time when basal phagocytic activity in control animals attains its circadian trough (Baciu et al. 1988). The same authors have shown that bilateral electrolytic lesions of the tubero-mammillary area (TM) are also followed by a severe depression of phagocytosis, and by the loss of its circadian oscillations, as well as by a suppression of the phagocytic response against bacterial antigens. This inhibition appears to last longer if the stimulation occurs during the light period. The functional relationship which exists between the TM area and immunity was confirmed by the report by Forni et col. (1983) that radio frequency destruction of the hypothalamic tubero-infundibular region permanently abrogates NK cell activity in mice.

Felegean et al. (1997) demonstrated that the arcuate nucleus, located in the TM area, is particularly involved in the phagocytic response in rats, showing that limited lesions of this structure lower the phagocytic activity subsequent to an antigenic challenge. Our experimental results (Hriscu et al. 1997) confirmed that triggering of the phagocytic response is controlled by the arcuate nucleus, sinceits quasi-complete destruction by means of neonatal monosodium glutamate treatment (Belluardo et al. 1990) practically suppresses the process. The arcuate nucleus, whose chemical damage affects the basal phagocytic activity only moderately, might however control its circadian rhythm. This hypothesis is supported by the existence of bi-directional connections between neuronal subpopulations in the SCN and in the arcuate nucleus, respectively, which express a variable tonus over the light-dark cycle (Saeb-Parsy and Dyball 2003a,b). Also, the arcuate nucleus includes certain neuronal groups exhibiting intrinsic rhythmic activity, synchronised with, but not generated in, the SCN (Abe et al. 2002). 
The arcuate nucleus possesses abundant glutaminergic synapses, the glutamate was also identified as the major neurotransmitter in the retino-hypothalamic tract (Hastings 1997). Another structure with a rich glutaminergic innervation, the olfactory bulb, is also involved in maintaining basal phagocytic activity and its circadian rhythm (Saulea et al. 1998). The phagocytic rhythm in guinea pigs exposed to a 6:6 light-dark alternation tends to synchronise with the lighting regimen, displaying a second (much smaller) peak (Baciu et al. 1988). The above data suggest that circadian dynamics of phagocytosis and of the phagocytic response are due to a rhythmically oscillating tonus of the hypothalamic centres (anterior and preoptic hypothalamic area and tubero-mammillary area), which seem to be subjected to photic (the light-dark cycle) and olfactory modulatory influences.

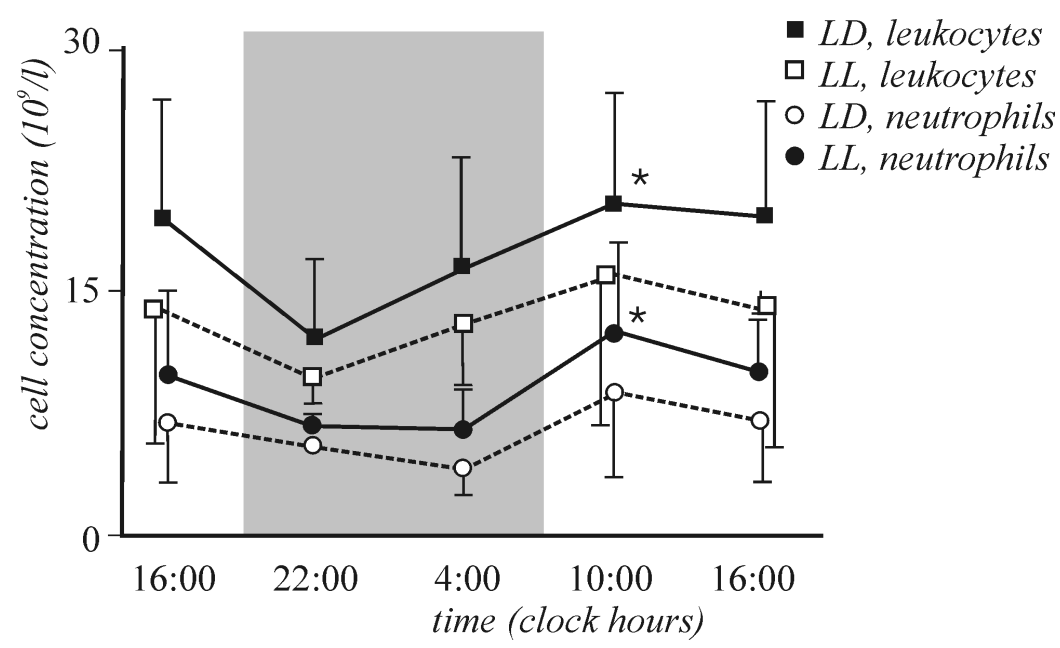

Fig. 7. Circadian variation of the leukocyte and neutrophil count in rats under $12 \mathrm{~h} / 12 \mathrm{~h}$ light-dark regime (LD) and constant light $(\mathrm{LL}){ }^{*}$ statistically significant $(\mathrm{p}<0.05)$ as compared with the lowest value

Many experimental data afford evidence that basal phagocytosis lies under the control of the anterior endocrinotropic hypothalamus. Electrolytic damage to the AHA is followed by a severe decrease in neutrophil enzymes (Saulea 1999), while its destruction in guinea pigs induces changes in phagocytosis that are very similar to the ones obtained by us with the functional pinealectomy provided by the constant light regimen.

AHA exerts its action via the thymus. Electrolytic lesions to the anterior hypothalamus in mice determine a rise of the plasma glucocorticoids, a rapid and severe involution of the thymus and a decrease in peripheral leukocytes (Pierpaoli and Yi 1990, Lesnikov and Tsvetkova 1985, Lesnikov et al. 1989); effects which melatonin is able to counteract (Maestroni and Conti 1990, 1996). Experimental thymectomy was followed by a decrease of about $30 \%$ of basal phagocytosis (Baciu et al. 1996). Also, AHA is one of the cerebral areas with relatively abundant melatonin receptors (Williams et al. 1995). Corroborating these data, our results allow us to hypothesise that pineal melatonin exerts its stimulatory effects on the basal phagocytic activity via AHA, which acts mainly by enhancing the thymic release of certain phagocytosis-activating peptides. A further argument for the idea of an
AHA-mediation of the effects of light is the fact that long lasting constant light produces morphologic alterations of the neurons in several hypothalamic regions, including the preoptic area of the anterior hypothalamus (Lin et al. 1990).

In analysing the influence exerted by the lighting regimen upon neutrophil phagocytosis and adherence, two distinct aspects have to be considered: the circadian structure of the rhythms and the level of the assessed functions.

There are several indirect and also direct indicators that melatonin, secreted exclusively at night, would play a role in the neutrophil function; however there are many discrepancies. Indolic precursors, such as tryptophan and serotonin, exert a stimulatory action upon phagocytosis in unicellular organisms (Csaba 1993) and in mammalian macrophages (Sánchez et al. 2004). Melatonin is directly involved in the neutrophil metabolism, in vitro (Recchioni et al. 1998, Olsen et al. 2003). In vitro studies employing pharmacological doses of melatonin $(5-100 \mu \mathrm{M})$ revealed a dose-dependent activation of the phagocytic function of ring dove heterophils (Rodríguez et al. 1997). However, such doses are far above the physiologically available range. The same authors showed that the phagocytic capacity of Streptopelia heterophils isolated over a 24-h 
cycle parallels the serum level of melatonin, both being highest at night, at about 04:00 (Rodríguez et al. 1999). It was later shown that melatonin concentrations corresponding to the nocturnal physiological level also enhance the phagocytic activity of Streptopelia heterophils and decrease the superoxid anion levels (Rodríguez et al. 2001). Barriga et al. (2001) reported similar results in mice. Our data in rats, showing that phagocytosis is higher during the nocturnal melatonin surge and lower in animals kept under constant light, also suggest that melatonin would be physiologically involved in the phagocytic function.

Our experiments, showing that phagocytosis in rats exposed to constant light does not lose its rhythm, but its circadian amplitude increases, reject the hypothesis that the circadian dynamics of basal phagocytosis would depend on the nocturnal melatonin secretion, although in mice and rats the high levels of plasma melatonin are coincident with those of phagocytosis. We may speak, more plausibly, about an endogenous chronome, generated and maintained by the hypothalamic pacemaker and modulated by external influences. In the absence of the time cue represented by dark onset (and not by the melatonin surge, which in rats occurs several hours later), the rhythm tends to lose synchronisation, to free-run, what leads to an advance of the acrophase in LL rats.

There is very little experimental work regarding the adherence of neutrophils, and data concerning the influence of melatonin is particularly scarce. Rodríguez et al. (1997) found no modification of the nylon fibre adherence of Streptopelia heterophils incubated with melatonin, irrespective of the dose used. In vivo, Lotufo et al. (2001) showed that melatonin in a dose comparable to the physiologic nocturnal concentrations exerts an inhibitory action upon the rolling and adhesion of rat leukocytes. Our results also suggest that neutrophil adherence is inversely correlated with the circadian oscillation of plasma melatonin, being higher at day and lower at night. However, it should be considered that various pharmacologically active chemicals, such as sodium salicilate and hydrocortisone succinate, have a different effect on neutrophil adhesion in vitro and in vivo, being able to inhibit adhesion in vivo, but not in vitro (MacGregor et al. 1974). Generally speaking, melatonin is considered to play an anti-inflammatory role, enhancing apoptosis of neutrophils (Chen et al. 2003), inhibiting their adhesion to endothelia and reducing PMN infiltration into damaged tissues (Reiter et al. 1998, Cuzzocrea et al. 1997), effects mediated by specific receptors (Dubocovich et al. 2003). Our data, showing that constant light induces a certain degree of inhibition to PMN adhesion, resulting both in a decreased amplitude and in a lower circadian average of the rhythm, suggests that endogenous melatonin is needed in order to maintain the characteristics of basal neutrophil adherence.

The ability to adhere of neutrophils in the blood of rats exhibits a circadian rhythm with two peaks, a mean peak at 10:00 and a second one, of lower amplitude, at 22:00. This time structure is very similar to that described by Saeb-Parsy (2003a) for a specific neuronal subpopulation in the $\mathrm{SCN}$ with projections to the arcuate nucleus and/or the supraoptic region. The activity of this group of neurons has two maxima, close to the light-dark (circadian time 10-12) and dark-light (circadian time 22-24) transitions, respectively. While the phase-relation between these rhythms does by no means necessarily imply a causal relationship, it might however hint at control of the adherenceprocess by this specific neuronal group. Moreover, the ML1 receptors mRNA expression exhibits a robust circadian rhythm with two maxima with a very similar time disposition (Poirel et al. 2002). This complex system, melatonin-SCNarcuate nucleus, could account for the biphasic rhythm of neutrophil adherence.

Inhibition of the adherence capacity of neutrophils is probably attributable, at least in part, to the high level of plasma cortisol, rather than to the absence of melatonin. Is is well-known that constant light activates the hypothalamichypophyseal-adrenal axis, stimulating cortisol secretion. Cortisol, prednisone, as well as other steroidic and non-steroidic anti-inflammatory drugs, possess a proven inhibitory effect upon neutrophil adhesion in vivo, part of their pharmacological action residing precisely on this effect (MacGregor et al. 1974).

Adhesion appears to be synchronous not with the rhythm of phagocytosis properly, but with that of other components of the inflammatory process, such as vascular permeability (higher at midday than at midnight - Lopez et al. 1997) and chemotactic migration of granulocytes, which is also higher in the morning hours (Bureau et al. 1991, Garelly et al. 1991). These data suggest that the mechanisms controlling basal phagocytosis are different from those which intervene during the inflammatory reaction, and that adhesion of neutrophils to endothelial cells accomplishes a functional role beyond being just the first step of the phagocytic process.

Our experimental results indicate that melatonin acts in a stimulatory manner with regard to the leukocyte populations in physiological conditions. Rats kept under constant light show a decreased mean circadian count of both neutrophils and lymphocytes, a fact in accordance with the known stimulatory effects of melatonin on the lymphocyte population in vitro: melatonin enhances proliferation of splenocytes and activates the release of IL-1 $\beta$ and IL-2 (Arias et al. 2003). The number of circulating $\mathrm{B}$ lymphocytes correlates 
positively with a short photoperiod, thus also with high levels of plasma melatonin (Yellon and Tan 2002), and also with circulating melatonin levels in healthy human subjects (Akbulut et al. 1999).

Taking the above together, we may conclude that not only the number, but also the basal and reactive phagocytic activity and the adherence of neutrophils are eminently periodic functions. Their innate rhythms, part of the circadian oscillation ofthe whole immune system, are susceptible to circadian modulation under the impact of endogenous and exogenous factors, among which are melatonin and the light-dark cycle. Based on our data, as well as on that of other authors, we propose a functional scheme integrating the central mechanisms with photic afferences which modulate neutrophil activity (Fig. 8). In this scheme, some links have been certified, while other are still hypothetical, and await their experimental confirmation.

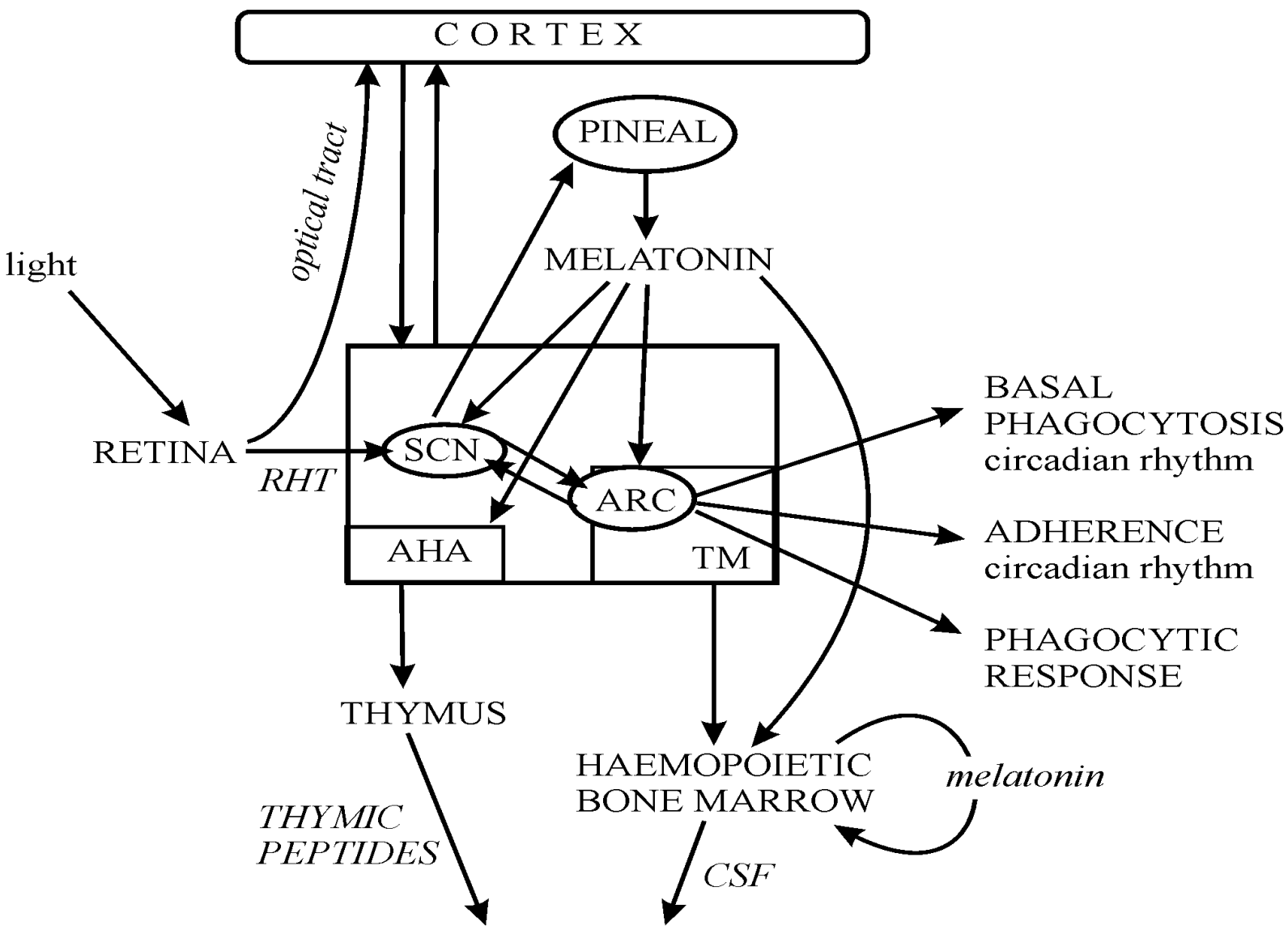

BASAL PHAGOCYTOSIS

Fig. 8. Integrative scheme of the central mechanisms modulating circadian neutrophil activity. RHT=retinohypothalamic tract, $\mathrm{SCN}=$ suprachiasmatic nucleus, $\mathrm{AHA}=$ anterior hyopthalamic area, $\mathrm{TM}=$ tubero-mammillary area, $\mathrm{ARC}=$ arcuate nucleus, $\mathrm{CSF}=$ colony-stimulating factor

\section{ACKNOWLEDGEMENTS}

I gratefully acknowledge the contribution of Germaine Cornélissen-Guillaume, $\mathrm{MD}, \mathrm{PhD}$, and F. Halberg, MD (Halberg Chronobiology Center of the University of Minnesota, USA), who performed the single cosinor rhythmometry of the data series showed herein.

\section{REFERENCES}

Abe M., E.D. Herzog, S. Yamazaki, M. Straume, H. Tei, Y. Sakaki, M. Menaker, G.D. Block: Circadian rhythms in isolated brain regions. J. Neurosci. 22: 350-356, 2002.

Akbulut H., F. Icli, A. Buyukcelik, K.G. Akbulut, S. Demirci: The role of granulocyte- 
macrophage-colony stimulating factor, cortisol, and melatonin in the regulation of the circadian rhythms of peripheral blood cells in healthy volunteers and patients with breast cancer. J. Pineal Res. 26: 1-8, 1999.

Angeli A., G. Gatti, M.L. Sartori, D. Del Ponte, R. Cerignola: Effects of exogenous melatonin on human natural killer (NK) cells activity. An approach to the immunomodulatory role of the pineal gland. In Gupta D., A.Attanasio, R.J. Reiter (eds.): The pineal gland and cancer. 1988, pp. 145-147.

Arendt J.: Melatonin and the Mammalian Pineal Gland. Chapman and Hall, London, 1995.

Arendt J.: Role of the pineal gland and melatonin in seasonal reproductive function in mammals. Oxford Reviews of Reproductive Biology 8: 266-320, 1986.

Arias J., E. Melean, N. Valero, H. Pons, L. Chacin-Bonilla, Y. Larreal, E. Bonilla: Effect of melatonin on lymphocyte proliferation and production of interleukin-2 (IL-2) and interleukin-1 beta (IL-1 beta) in mice splenocytes (In Spanish). Invest. Clin. 44: 4150, 2003.

Baciu I., G. Benetato, S. Secăreanu: L'influence des fractions globuliniques du sérum, isolées par chromatographie sur colonne à derivés céllulosiques, sur la phagocytose. 5eme Congres Mondial de Biochimie, Moscou, 1961.

Baciu I., A. Olteanu, T. Prodan, M. Baiescu, A. Vaida: Changes of phagocytic biological rhythm by reduction of circadian times and by influences upon hypothalamus. Int. J. Neurosci. 41: 143-153, 1988.

Baciu I., C. Cornélissen, A. Olteanu, F. Halberg: Chrono-meta-analysis of circadian phagocytosis rhythms in blood of ginea pigs on two different lighting regimens. Chronobiologia 21: 299-302, 1994.

Baciu I., A. Felegean, T. Maghiar, C. Hozan, M. Albu: Effects of thymectomy on blood neutrophils phagocytic activity and phagocytic response in mice. Rom. J. Physiol. 33: 75-81, 1996.

Baciu I., M. Hriscu, G. Saulea: Hypothalamic mechanisms of immunity. Intern. J. Neurosci. 113: 259-277, 2003.

Barjavel M.J., Z. Mamdouh, N. Raghbate, O. Bakouche: Differential expression of the melatonin receptor in human monocytes. J. Immunol. 160: 1191-1197, 1988.

Barriga C., M.I. Martín, R. Tabla, E. Ortega, A.B. Rodríguez: Circadian rhythm of melatonin corticosterone and phagocytosis: effect of stress. J. Pineal Res. 30: 180-187, 2001.

Belluardo N., G. Mudo', M. Bindoni: Effects of early destruction of the mouse arcuate nucleus by monosodium glutamate on age-dependent natural killer activity. Brain Res. 534: 225-233, 1990.

Benetato G., I. Baciu, L. Vlad: Zentralnervensystem und Abwehrfunction. Die Rolle der hypothalamischen vegetativen Zentren bei der Phagozytentätigkeit. Schweiz. Med. Wochenschr. 75: 702-712, 1945.

Benetato Gr., C. Oprişiu, I. Baciu: Système nerveux central et phagocytose (contributions expérimentales grâce à la méthode de la "tête isolée"). J. Physiol. 39: 191-197, 1947.

Berger J.: Changes in marrow myelopoietic and lymphoid cell counts after repeated cyclophosphamide administration in rats. Haematologia 14: 407-416, 1981.

Berger J. and M. Slapničková: Circadian structure of rat neutrophil phagocytosis. Comp. Clin. Path. 12: 84-89, 2003.

Berk M.L. and J.A. Finkelstein: An autoradiographic determination of the efferent projections of the suprachiasmatic nucleus of the hypothalamus. Brain Res. 226: 1-13, 1981.

Bongrand P., G. Bouvenot, R. Bartolin, J. Tatossian, B. Bruguerolle: Are there circadian variations of polymorphonuclear phagocytosis in man? Chronobiol. Int. 5: 81-83, 1988.

Bureau J.P., M. Coupe, G. Labreque, P. Vago: Chronobiologie de l'inflammation. Path. Biol. 35: 942-950, 1987.

Bureau J.P., L. Garelly, P. Vago: Nycthemeral variations on LPS- and BCG-induced PMN migration in normal mice. Int. J. Tissue React. 13: 203-206, 1991.

Chen J.C., C.J. Ng, T.F. Chiu, H.M. Chen: Altered neutrophil apoptosis activity is reversed by melatonin in liver ischemia-reperfusion. J. Pineal Res. 34: 260-264, 2003.

Conti A., G.J.M. Maestroni: Melatonin-induced immuno-opioids: role in lymphoproliferative and autioimmune diseases. Adv. Pineal Res. 7: 83-100, 1994.

Cornélissen G., F. Halberg: Introduction to chronobiology. Medtronic chronobiology Seminar No. 7, Library of Congress, Catalog Card No. 94-060580, 1994.

Csaba G.: Presence in and effects of pineal indoleamines at very low level of phylogeny. Experientia 49: 627-634, 1993.

Cuzzocrea S., B. Zingarelli, E. Gilad, P. Hake, A.L. Salzman, C. Szabo: Protective effect of melatonin in carrageenan-induced models of local inflammation: relationship to its inhibitory effect on nitric oxide production and its peroxynitrite scavenging activity. J. Pineal Res. 23: 106-116, 1997.

Doherty D.E., C. Haslett, M.G. Fonnesen, P.M. Henson: Human monocyte adherence: a primary effect of chemotactic factors on the monocyte to stimulate adherence to human 
endothelium. J. Immunol. 138: 1762-1771, 1987.

Dubocovich M.L., M.A. Rivera-Bermudez, M.J. Gerdin, M.L. Masana: Molecular pharmacology regulation and function of mammalian melatonin receptors. Front. Biosci. 8d: 1093-1108, 2003.

Felegean A., M. Junie, I. Baciu: Effects of the electrolytic damage of the arcuate nucleus on the blood neutrophils phagocytic activity and phagocytic response. Rom. J. Physiol. 34: 7582, 1997.

Forni G., M. Bindoni, A. Santoni, N. Belluardo, A.E. Marchese, M. Giovarelli: Radio frequency destruction of the tubero-infundibular region of hypothalamus permanently abrogates NK cell activity in mice. Nature 306: 181-185, 1983.

Garelly L., J.P. Bureau, G. Labreque: Temporal study of carrageenan-induced PMN migration in mice. Agents Actions 33: 225-228, 1991.

Gatti G., R.G. Masera, R. Carignola, M.L. Sartori, E. Margro, A. Angeli. Circadian-singledependent enhancement of human natural killer cell activity by melatonin. J. Immunol. Res. 2: 108-116, 1990.

Halberg F.: Temporal coordination of physiological function. Cold Spr. Harb. Symp. Quant. Biol. 25: 289-310, 1960.

Hastings M.H.: The vertebrate clock: localisation connection and entrainment. In Redfern P.H. and B. Lemmer (eds.): Physiology and Pharmacology of Biological Rhythms. Springer 1997, pp. 1-21.

Haus E.: Biologic rhythms in hematology. Pathol. Biol. 44 (Paris): 618-630, 1996.

Hriscu M.: Modulatory factors of circadian phagocytic activity. Doctoral thesis, BabeşBolyai University, Cluj-Napoca, 2003.

Hriscu M. and G. Saulea: Phagocytic activity and adherence of neutrophils in rats under light-dark alternation and constant light. Physiology National Conference, Cluj-Napoca 2002.

Hriscu M., G. Saulea, N. Vidraşcu, I. Baciu: Effects of monosodium glutamate on blood neutrophils phagocytic activity and phagocytic response in mice. Rom. J. Physiol. 34: 95-101, 1997.

Hriscu M., G. Saulea, N. Vidraşcu, I. Baciu: Circadian rhythm of phagocytosis in mice. Rom. J. Physiol. 35: 315-319, 1998.

Illnerova H. and J. Vanecek: Response of rat pineal serotonin $\mathrm{N}$-acetyltransferase to one minute light pulse at different hight times. Brain Res. 167: 431-434, 1979.

Klein D.C. and J.L. Weller: Indole metabolism in the pineal gland: a circadian rhythm in $\mathrm{N}$-acetyltransferase. Science 169: 1093-1095, 1970.
Lesnikov V.A. and I.P. Cvetkova: Stereotaxic coordinates of the hypothalamus in mice (In Russian). Fiziol. Zurn. 71: 798-804, 1985.

Lesnikov V.A., S.B. Adjieva, E.A. Korneva: Endogenous splenic colony-formation in mice in experimental pathology of the CNS (In Russian). Patol. Fiziol. 6: 14-17, 1989.

Lin Y.L., M.Y. Tai, Y.F. Tsai: Morphological changes in the hypothalamic neurons of female rats exposed to continuous illumination. Chin. J. Physiol. 33: 291-298, 1990.

Lopez C., J.L. deLyra, R. Pekelmann Markus, M. Mariano: Circadian rhythm in experimental granulomatous inflammation is modulated by melatonin. J. Pineal Res. 23: 72-78, 1997.

Lotufo C.M., C. Lopes, M.L. Dubocovich, S.H. Farsky, L.P. Markus: Melatonin and Nacetylserotonin inhibit leukocyte rolling and adhesion to rat microcirculation. Eur. J. Pharmacol. 430: 351-357, 2001.

MacGregor R.R., P. Spagnuolo, A. Lentnek: Inhibition of granulocyte adherence by ethanol prednisone and aspirin measured with an assay system. N. Engl. J. Med.: 642-646, 1974.

MacGregor R.R., E. Macarak, N. Kefalides: Comparative adherence of granulocytes to endothelial monolayer and nylon fiber. 61: 697702, 1978.

Maestroni G.J.M. and A. Conti: The pineal neurohormone melatonin stimulates activated CD4+ Thy-1+ cells to release opioid agonists with immunoenhancing and anti-stress properties. J. Neuroimmunomodul. 28: 167176, 1990.

Maestroni G.J.M. and A. Conti: Melatonin and the immuno-hematopoietic system therapeutic and adverse pharmacological correlates. J. Neuroimmunomodul. 3: 325-332, 1996.

Maestroni G.J.M., A. Conti, W. Pierpaoli: Role of the pineal gland in immunity: Circadian synthesis and release of melatonin modulates the antibody response and antagonizes the immunosuppressive effect of corticosterone. J. Neuroimmunol. 13: 19-30, 1986.

Maestroni G.J.M., A. Conti, W. Pierpaoli: Role of the pineal gland in immunity: II. Melatonin enhances the antibody response via an opiatergic mechanism. Clin. Exp. Immunol. 68: 384-391, 1987.

Morrey K.M., J.A. McLachlan, C.D. Serkin, O. Bakouche: Activation of human monocytes by the pineal hormone melatonin. J. Immunol. 153: 2671-2680, 1994.

Olsen L.F., U. Kummer, A.L. Kindzelskii, H.R. Petty: A model of the oscillatory metabolism of activated neutrophils. Biophys. J. 84: 69-81, 2003.

Pickard G.E.: The afferent connections of the suprachiasmatic nucleus of the golden hamster 
with emphasis on the retinohypothalamic projection. J. Comp. Neurol. 211: 65-83, 1982.

Pickard G.E., F. W. Turek: The hypothalamic paraventricular nucleus mediates the photoperiodic control of reproduction but not the effects of light on the circadian rhythm of activity. Neurosci. Lett. 43: 67-72, 1983.

Pieri C., R. Recchioni, F. Moroni, F. Marcheselli, M. Marra, S. Marinoni, R. Di Primio: Melatonin regulates the respiratory burst of human neutrophils and their depolarization. J. Pineal Res. 24: 43-49, 1998.

Pierpaoli W. and C.X. Yi: The involvement of pineal gland in immunity and aging: I. Thymusmediated immunoreconstituting and antiviral activity of thyrotropin-releasing hormone. J. Neuroimmunol. 27: 99-109, 1990.

Poirel V.J., M. Masson-Pevet, P. Pevet, F. Gauer: MT1 melatonin receptor mRNA expression exhibits a circadian variation in the rat suprachiasmatic nuclei. Brain Res. 946: 64-71, 2002.

Pownall R., P.A. Kabler, M.S. Knapp: The time of day of antigen encounter influences the magnitude of the immune response. Clin. Exp. Immunol. 36: 347-354, 1979.

Recchioni R., F. Marcheselli, F. Moroni, R. Gaspar, S. Damjanovich, C. Pieri: Melatonin increases the intensity of respiratory burst and prevents L-selectin shedding in human neutrophils in vitro. Biochem. Biophys. Res. Commun. 252: 20-24, 1998.

Reiter R.J., J.J. Garcia, J. Pie: Oxidative toxicity in models of neurodegeneration: responses to melatonin. Restor. Neurol. Neurosci. 12: 135142, 1998.

Rodríguez A.B. and R.W. Lea: Effect of pinealectomy upon the nonspecific immune response of the ring dove (Streptopelia risoria). J. Pineal Res. 16: 159-166, 1994.

Rodríguez A.B., E. Ortega, R.W. Lea, C. Barriga: Melatonin and the phagocytic process of heterophils from the ring dove (Streptopelia risoria). Molec. Cell. Biochem. 168: 185-190, 1997.

Rodríguez A.B., J.M. Marchena, G. Nogales, J. Duran, C. Barriga: Correlation between the circadian rhythm of melatonin phagocytosis and superoxide anion levels in ring dove heterophils. J. Pineal Res. 26: 35-42, 1999.
Rodríguez A.B., P.M. Terron, J. Durán, E. Ortega, C. Barriga: Physiological concentrations of melatonin and corticosterone affect phagocytosis and oxidative metabolism of ring dove heterophils. J. Pineal Res. 31: 31-38, 2001.

Saeb-Parsy K. and R.E. Dyball: Defined cell groups in the rat suprachiasmatic nucleus have different day/night rhythms of single-unit activity in vivo. J. Biol. Rhythms 18: 26-42, 2003a.

Saeb-Parsy K. and R.E. Dyball: Responses of cells in the rat suprachiasmatic nucleus in vivo to stimulation of afferent pathways are different at different times of the light/dark cycle. J. Neuroendocrinol. 15: 895-903, 2003b.

Sainz R.M., J.C. Mayo, H. Uria, M. Kotler, I. Antolin, C. Rodriguez, A. Menendez-Pelaez: The pineal hormone melatonin prevents in vivo and in vitro apoptosis in thymocytes. J. Pineal Res. 19: 178-188, 1995.

Sánchez S., S.D. Paredes, M.I. Martín, C. Barrigua, A.B. Rodríguez: Effect of tryptophan administration on circulating levels of melatonin and phagocytic activity. J. Appl. Biomed. 2: 169-177, 2004.

Saulea G.: The olfactory bulb and immunity. Doctoral thesis, University of Medicine and Pharmacy, Cluj-Napoca 1999.

Saulea G., M. Hriscu, N. Vidraşcu, I. Baciu: Influence of bilateral olfactory bulbectomy on the circadian rhythm of phagocytic activity in mice. Rom. J. Physiol. 35: 309-314, 1998.

Swanson L.W. and W.M. Cowan: The efferent connections of the suprachiasmatic nucleus of the hypothalamus. J. Comp. Neurol. 160: 1-12, 1975.

Swoyer J., E. Haus, L. Sackett-Lundeen: Circadian reference values for hematologic parameters in several strains of mice. Prog. Clin. Biol. Res. 227 A: 281-296, 1987.

Vanecek J.: Cellular Mechanisms of Melatonin Action. Phys. Rev. 78: 687-721, 1998.

Williams L.M., L.T. Hannah, M.H. Hastings, E.S. Maywood: Melatonin receptors in the rat brain and pituitary. J. Pineal Res. 19: 173-177, 1995.

Yellon S.M. and L.T. Tran: Photoperiod reproduction and immunity in select strains of inbred mice. J. Biol. Rhythms 7: 65-67, 2002.

\section{Address:}

Monica L. Hriscu, Institute of Public Health, Occupational Medicine Dept., 6 Pasteur St., 400349 Cluj-Napoca, Romania; mhriscu@ispcj.ro 